\title{
Circumferential membrane-like aortic dissection with intimo-intimal intussusception
}

\author{
Ayca Boyaci, Ozcan Ozeke, Ahmet Saritas
}

Health Sciences University, Turkiye Yuksek Ihtisas Training and Research Hospital,Department of Cardiology, Ankara, Turkey

\section{ARTICLE INFO}

Article history:

Submitted: 21. 6. 2018

Accepted: 19. 9. 2018

Available online: 17. 9. 2019

\section{Klíčová slova:}

Cirkumferenciální aortální disekce Intimointimální intususcepce

\author{
Keywords: \\ Circumferential aortic dissection \\ Intimo-intimal intussusception
}

\begin{abstract}
Although the intimal tears in type $\mathrm{A}$ aortic dissections are usually transverse, the intimo-intimal intussusception is an unusual type of acute aortic dissection in which the intimal tear occurs circumferentially. 1,2 Aortography may be suboptimal given that a circumferential dissection flap may not be clearly seen. ${ }^{3}$ It can be also misdiagnosed as a perforated membranous type supravalvular aortic stenosis. ${ }^{1}$ A 65 -year-old man presented with sudden onset severe chest pain. On emergency bedside echocardiography, ascending aortic aneurysm with severe aortic regurgitation was reported without evidence of aortic dissection appearance. Aortic angiogram showed circumferential membrane-like dissection flap with severe aortic regurgitation (Fig. 1). At operation, a well-defined demarcation was noted and a circumferential intimal tear was identified. Aortic root replacement with the Yacoub/ David techniques were performed; the patient had an uncomplicated postoperative course.
\end{abstract}

\section{Appendix A. Supplementary data}

Supplementary data associated with this article can be found in the online version.

\section{References}

1. Dimarakis I, Yadav R, Bahia S, et al. Acute type A aortic dissection mimicking a congenital supravalvular aortic membrane. J Thorac Cardiovasc Surg 2007;134:1340-1341.

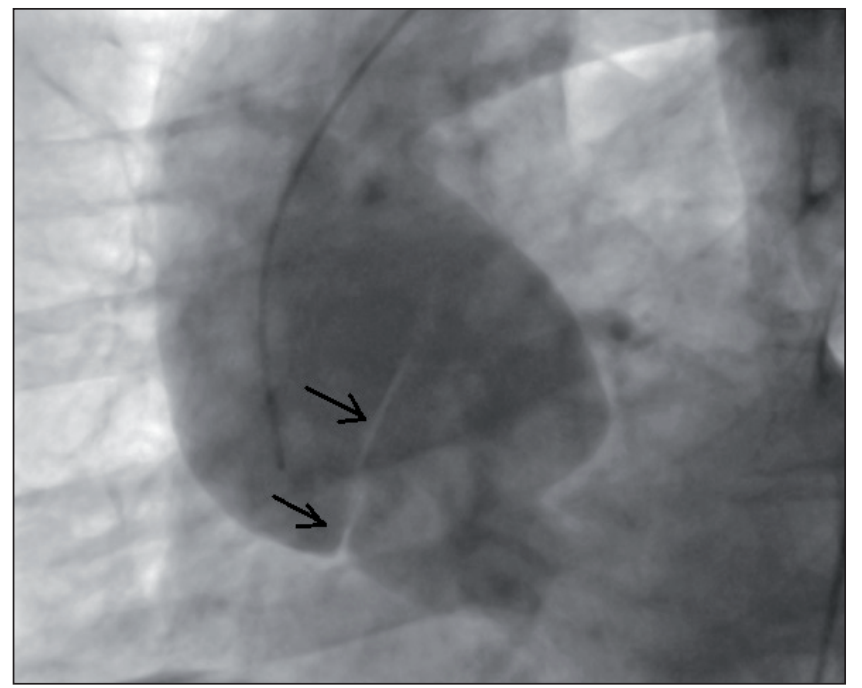

Fig. 1 - Aortic angiogram shows a circumferential membrane-like dissection flap (arrows).

2. Shah PJ, Singh SS, Chaloob SS, et al. Intimo-intimal intussusception of the aorta. Ann Thorac Surg 2006;82:2274-2276.

3. Ozeke O, Maden O, Selcuk MT, et al. Pseudo-double aortic valve imaging caused by type A aortic dissection. J Cardiovasc Med 2007;8:760. 